\title{
Three-dimensional imaging with reflection synthetic confocal microscopy
}

\author{
Md Rasedujuaman ${ }^{1}$, Kévin Affannoukoué ${ }^{1}$, Nicolas Garcia-Seyda ${ }^{2}$, Philippe Robert ${ }^{2}$, \\ Hugues Giovannini ${ }^{1}$, Patrick C. Chaumet ${ }^{1}$, Olivier Theodoly ${ }^{2}$, Marie-Pierre Valignat ${ }^{2}$, \\ Kamal Belkebir ${ }^{1}$, Anne Sentenac ${ }^{1}$, And Guillaume Maire ${ }^{1, *}$ \\ ${ }^{1}$ Aix Marseille Univ, CNRS, Centrale Marseille, Institut Fresnel, Marseille, France \\ ${ }^{2}$ Laboratoire Adhesion et Inflammation, UMR INSERM 1067, UMR CNRS 7333, Aix-Marseille Université, Assistance Publique-Hôpitaux de Marseille, Case \\ 937, 13288 Marseille Cedex 09, France \\ ${ }^{*}$ Corresponding author: guillaume.maire@fresnel.fr
}

Compiled May 29, 2020

\begin{abstract}
Biomedical imaging lacks label free microscopy techniques able to reconstruct in $3 \mathrm{D}$ and with high resolution the contour of biological cells in solution, as required for the fast diagnosis of numerous diseases. Inspired by computational OCT techniques, we present a tomographic diffractive microscope in reflection geometry used as a synthetic confocal microscope, compatible with this goal and validated with the $3 \mathrm{D}$ reconstruction of a human effector T lymphocyte. ( 2020 Optical Society of America
\end{abstract}

http://dx.doi.org/10.1364/ao.XX.XXXXXX

\section{INTRODUCTION}

Optical diffraction microscopy is an important tool in biological and biomedical imaging as it can be used on live cells and does not require staining. Yet, its poor axial resolution compared to the transverse one limits its interest for three-dimensional imaging. Now, an increasing number of applications would benefit from highly resolved 3D images of cells. In particular, the ability to observe the contour deformation of two interacting cells is of major interest as conformational changes can be the precursor of important biological phenomena [1].

Presently, the best resolved marker-free 3D images of cells have been obtained using computational Tomographic Diffraction Microscopy (TDM). It consists in reconstructing digitally the sample contrast from a stack of holograms obtained by interferometry under different illuminations (usually provided by a monochromatic collimated beam with varying angles) [2]. With such a data set, it is possible to form a 3D image with a resolution typically twice better than the standard microscopy techniques.

However, most of the studies in TDM have been performed with set-ups in transmission [2-8], which eases the volume reconstruction of the sample, but ends up with an axial resolution remaining at least three times worse than the transverse one. As a result, 3D conformation changes at cell membranes or interfaces in the wavelength range cannot be resolved.
To image the cell contour in $3 \mathrm{D}$, the reflection geometry, which is highly sensitive to reflections from interfaces but not to slowly varying volume inhomogeneities, may be more appropriate [9]. In biomedical imaging, this geometry is mainly encountered in Optical Coherence Tomography (OCT) [10], where the axial resolution, of at best one micron, remains insufficient to detect sub-micrometer axial deformation such as those encountered in lymphocyte activation [1]. In this context, reflection TDM, with its theoretical twice better resolution [11], seems an interesting solution.

So far, this computational approach has been applied to image thin structures deposited or etched on a reflective substrate [1216], and to obtain 2D reflectance images of cells [? ]. Combined with broad band illumination, it was also used to image reflective targets under a thick diffusive layer $[17,18]$ and biological tissues at different depths $[19,20]$, the digital reconstruction allowing an efficient removal of the multiple scattering and aberrations deteriorating the images of standard OCT.

In this paper, we apply reflection TDM to the 3D imaging of cells. We show how a TDM set-up can be used as a synthetic confocal microscope (SyCM) and we take advantage of this computational approach to correct the focus aberrations induced by the use of a high numerical aperture oil-immersion objective $(\mathrm{NA}=1.49)$. We compare reflection and transmission TDM on simulated data and provide experimental images of calibrated and biological samples.

\section{LINKS BETWEEN TOMOGRAPHIC DIFFRACTIVE MI- CROSCOPY AND CONFOCAL MICROSCOPY}

TDM permits to retrieve the 3D map of refractive index of a sample from the measurement of its scattered field under various illumination angles, using a numerical reconstruction procedure. A sensitivity to refractive index contrasts below 0.01 is typically attained [5-8]. Usually the field is measured in a plane conjugated to the sample, and then transferred to the far-field (Fourier space) with a 2D discrete Fourier transform to ease the data treatment steps.

The simplest link between the sample refractive index map and the scattered field is obtained under the Born approxima- 
tion, typically valid for samples with weak refractive index contrast [2]. In this case, in the scalar approximation, the field scattered in far-field along wave vector $\mathbf{k}_{s}$ for an illumination plane wave along wave vector $\mathbf{k}_{i}$ is given by

$$
E_{S}\left(\mathbf{k}_{s}, \mathbf{k}_{i}\right) \propto \tilde{\Delta \varepsilon}\left(\mathbf{k}_{s}-\mathbf{k}_{i}\right),
$$

where $\tilde{\Delta \varepsilon}$ is the 3D Fourier transform of the permitivitty contrast $\Delta \varepsilon$ of the sample (the permittivity being the refractive index squared). The reconstructed permittivity contrast $\Delta \varepsilon_{\text {rec }}$ at position $\mathbf{r}$ is thus directly obtained by a 3D discrete inverse Fourier transform :

$$
\Delta \varepsilon_{\text {rec }}(\mathbf{r}) \propto \sum_{\mathbf{k}_{i}} \sum_{\mathbf{k}_{s}} E_{S}\left(\mathbf{k}_{s}, \mathbf{k}_{i}\right) e^{i\left(\mathbf{k}_{s}-\mathbf{k}_{i}\right) \cdot \mathbf{r}}
$$

Such a procedure is also known as synthetic aperture generation: each illumination angle permits to access different Fourier components of the object, and combining them enlarges the accessible domain and improves the resolution.

Synthetic aperture generation is in fact equivalent to phase confocal imaging in which a beam is focused inside the sample and the scattered field is detected (with an interferometric set-up, as in OCT) at the conjugated position, in the image plane, of the focus position [18].

This equivalence can be understood by recalling that scattering is a linear process, the field scattered by a sample illuminated by a beam made of a sum of plane waves is equal to the sum of the scattered fields for each plane wave. Hence, assuming that the phase of all the incident plane waves is 0 at the focal point of the objective, corresponding to the origin of the coordinate system $\mathbf{r}=\mathbf{0}$,

$$
\tilde{e}\left(\mathbf{k}_{s}, \mathbf{r}\right)=\sum_{\mathbf{k}_{i}} E_{S}\left(\mathbf{k}_{s}, \mathbf{k}_{i}\right) e^{-i \mathbf{k}_{i} \cdot \mathbf{r}}
$$

represents the far field scattered in the $\mathbf{k}_{s}$ direction obtained when the sample is illuminated by a beam made of a sum of plane waves interfering constructively at $\mathbf{r}$. Then,

$$
E(\mathbf{r})=\sum_{\mathbf{k}_{s}} \tilde{e}\left(\mathbf{k}_{s}, \mathbf{r}\right) e^{i \mathbf{k}_{s} \cdot \mathbf{r}},
$$

represents the field at $\mathbf{r}$ in the image domain of the microscope (with magnification 1) obtained from the far-field $\tilde{e}$ [21]. It corresponds to the complex field that would be measured at the center of the pinhole of a confocal microscope. Introducing Eq. (3) in Eq. (4), one observes that the complex field $E$ is proportional to $\Delta \varepsilon_{\text {rec }}$ under Born approximation,

$$
E(\mathbf{r})=\sum_{\mathbf{k}_{i}} \sum_{\mathbf{k}_{s}} E_{S}\left(\mathbf{k}_{s}, \mathbf{k}_{i}\right) e^{i\left(\mathbf{k}_{s}-\mathbf{k}_{i}\right) \cdot \mathbf{r}} \propto \Delta \varepsilon_{\text {rec }}(\mathbf{r}) .
$$

Works in optical imaging based on synthetic aperture usually calculate the real and imaginary parts of Eq. (5) to obtain the refractive index and absorption maps of their samples. In fact calculating its intensity and phase is also meaningful, as they represent the squared modulus and the argument of the reflectance (or transmittance) of a focused beam scanned through the sample. We call this approach synthetic confocal microscopy (SyCM), which we consider more general than synthetic aperture generation, since optimized phase or amplitude terms can be inserted in Eqs. (3) and (4) to reshape numerically the illumination and detection of the microscope, as is done with Spatial Light Modulators in adaptive optics, and proposed in smartOCT [18]. In particular, this computational approach allows an easy correction of the focus aberrations induced by the index mismatch at the glass-water interface of our experimental configuration, where an oil-immersion microscope objective of $\mathrm{NA}=1.49$ is used for maximizing the axial and transverse resolution. This procedure will be detailed in part 4 .

\section{NUMERICAL RESULTS}

To show the interest of reflection SyCM, we first apply it to synthetic 3D objects: two portions of spheres of refractive index 1.38 in water. The axial cuts of the samples are depicted in Figs. 1(a) and (b). In Fig. 1(a) the top sphere touches the bottom one, whereas in Fig. 1(b) it enters the bottom one. These two configurations illustrate, with simple geometries, a T cell (top sphere) interacting with an antigen presenting cell (bottom sphere): in (a) the T cell is not activated, whereas in (b) it is activated [1].
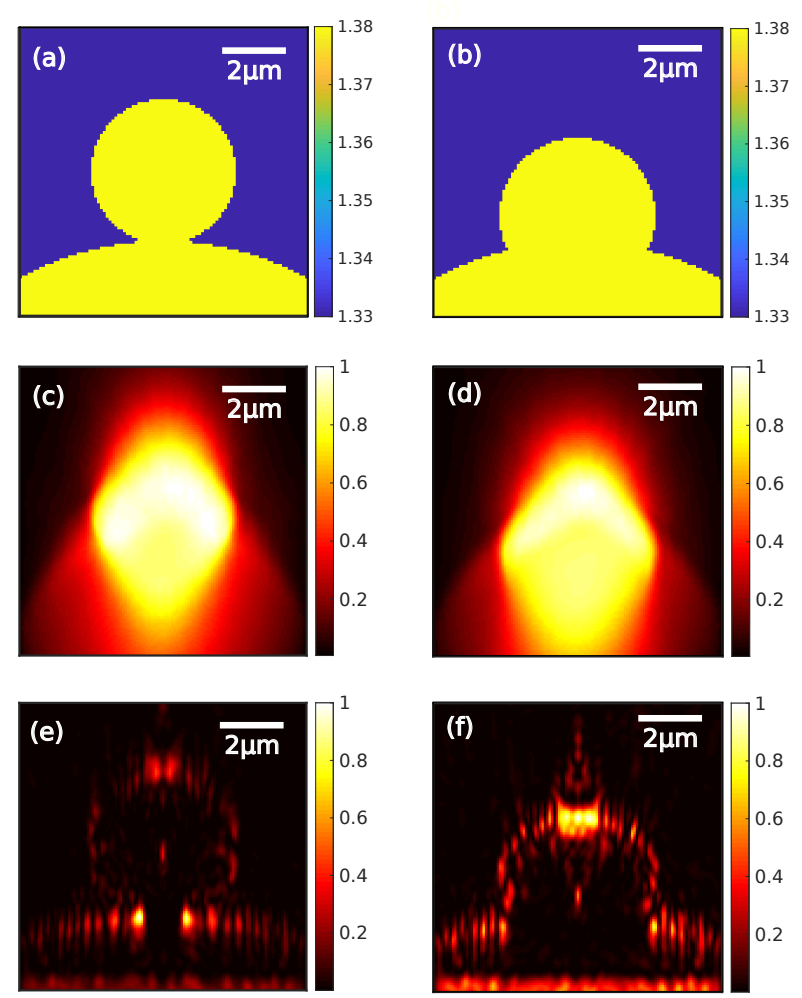

Fig. 1. (a) and (b): Axial cuts of two configurations mimicking two interacting cells (refractive index in colorbar). (c) and (d): respective SyCM intensity reconstructions in transmission geometry; (e) and (f) in reflection geometry. Reconstructions are normalized to 1 at their maximal value.

A home made program based on the Coupled Dipole Method (CDM) [22] estimated the scattered fields $E_{S}\left(\mathbf{k}_{s}, \mathbf{k}_{i}\right)$ of these two configurations by solving rigorously Maxwell equations. 121 linearly polarized plane waves were used for the illumination, with maximal polar angle of $55^{\circ}$, and a numerical aperture of 1.2 in water was assumed for detection. We chose an imaging configuration in which the incident and scattered transverse wave vectors laid on a regular two-dimensional grid. Calculations were performed in transmission and reflection. 
The 3D intensity $|E(\mathbf{r})|^{2}$, Eq. (5), was estimated plane by plane using 2D inverse Fourier transforms, as in [23]. In Figs. 1(c) and (d) the reconstructions obtained in transmission show no difference between the two cell-cell configurations. In contrast, the contour of the objects can be retrieved with high fidelity with data in reflection (Figs. 1(e) and (f)). This highlights the great potential of reflection SyCM to probe the surfaces and interfaces of biological cells.

\section{EXPERIMENTAL VALIDATION}

\section{A. Experimental set-up}

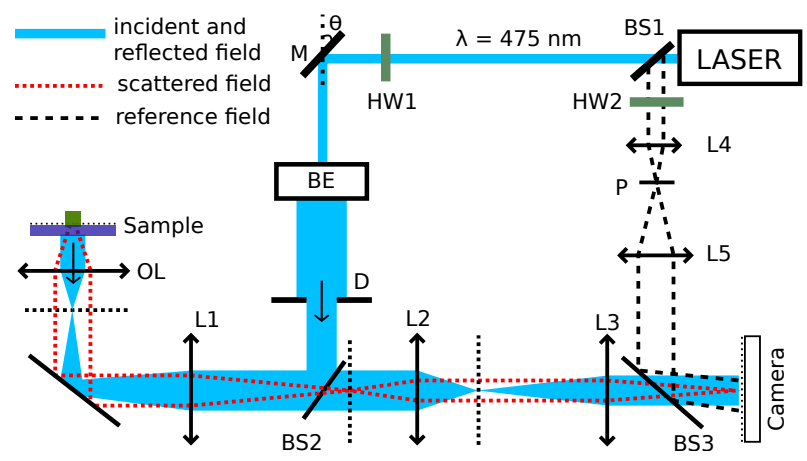

Fig. 2. Reflection TDM set-up: $M$, rotating mirror; $B E$, beam expander; D, diaphragm; OL, objective lens; $L_{1}$, tube-lens; $L_{2-5}$, lenses; $B S_{1-3}$, beamsplitters; $H W_{1-2}$, half-waveplates; $\mathrm{P}$, pinhole

We built a TDM set-up in reflection geometry sketched on Fig. 2. Detailed description can be found elsewhere [15]. The light source is a supercontinuum laser (NKT Photonics SuperK Extreme EXW-12) filtered at $475 \mathrm{~nm}$ with a spectral width of $6 \mathrm{~nm}$ thanks to a variable bandpass filter (NKT Photonics SuperK Varia). The laser beam is linearly polarized, its illumination angle controlled by a fast steering mirror (M, Newport FSM-300). The scattered field is collected by an oil-immersion objective (Nikon-TIRF 100X, NA = 1.49) and imaged on a sCMOS camera (Andor Zyla) with a global magnification of about 200.

For samples like biological cells in water, the back-scattered signal is very weak. Depending on the illumination angle, parasitic reflections from the tube lens and the objective can be of the same order as the useful signal. The use of a low coherence source for the off-axis holography permits to wash away these parasitic reflections: here, the coherence length is about $40 \mu \mathrm{m}$, much shorter than the distance between the sample and the parasitic reflective surfaces.

\section{B. Phase normalization procedure of the data set}

All the reconstruction procedures in TDM consider that the different incident plane waves used to illuminate the sample have the same null phase at the chosen origin of the coordinate system. This origin is usually taken at the focal point of the objective. But the mechanical and thermal drifts between successive illuminations introduce random phase shifts to each incident plane wave. A phase normalization procedure is therefore crucial to correct the data so that the phases of each illumination plane wave can be considered null at the origin. Then, forming a synthetic beam that focuses at any position is simple algebra.

This normalization is based on the complex amplitude of the far field in the specular reflected direction for each hologram. It corresponds to the reflection of the incident beam at the glasswater interface and appears as a Dirac-like peak in the Fourier plane of the microscope. We assume that this reflection is not affected by the sample. The whole signal is corrected so that the experimental specular reflection corresponds to its theoretical value. Contrarily to the transmission coefficient which is generally always close to one, the theoretical reflection coefficient can be easily calculated only if the glass-water interface is placed at the object focal plane (conjugated to the camera plane): it will then be equal to the Fresnel formula. However it is seldom the case, especially if the sample is thick. In this case, the optical path of the specular reflection towards the camera, and therefore the phase of the theoretical reflection coefficient, will strongly depend on the unknown distance $d$ between these two planes and on the illumination angle. To avoid this issue, we numerically propagate the fields to reach the glass-water interface. $d$ is estimated from a three dimensional image that is insensitive to illumination phase errors and corresponds to a 3D incoherent brightfield image:

$$
I(\mathbf{r})=\sum_{\mathbf{k}_{i}}\left|\sum_{\mathbf{k}_{s}} E_{S}\left(\mathbf{k}_{S}, \mathbf{k}_{i}\right) e^{i \mathbf{k}_{s} \cdot \mathbf{r}}\right|^{2} .
$$

Even though less resolved than the synthetic confocal reconstruction, the $3 \mathrm{D}$ brightfield image $I$ permits to detect the interface with enough accuracy for the phase normalization to be satisfactory.

\section{Experimental reconstructions}

SyCM was tested experimentally on a calibrated sample made of a glass bead of diameter $5 \mu \mathrm{m}$ and refractive index 1.46 (Bangs laboratories), deposited on a glass lamella and immersed in water. Figure 3 shows reconstructions obtained from simulated (a) and experimental (c) data. We observe that they are very similar, the slight differences can be attributed to discrepancies between the optical transfer function of the objective and the ideal one assumed for the simulations. Figure 3(b) shows the distorted reconstruction obtained when the phase normalization is done without propagating the fields to the glass-water interface. It points out the importance of this procedure, even for small values of $d$ (which, in this case, was estimated to $1.1 \mu \mathrm{m}$ ).
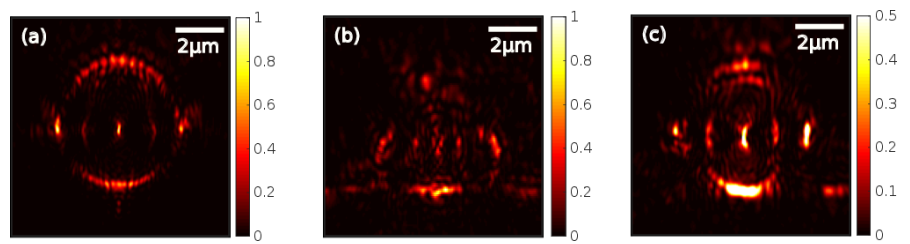

Fig. 3. Axial cuts of SyCM intensity reconstructions for a glass bead immersed in water: synthetic data (a), experimental data without (b) and with (c) the correct phase normalization. Colorscale has been readjusted on (c) for visualization purpose.

Note that the reconstructions are performed in darkfield mode, by filtering out the specular reflection in the Fourier space for each hologram. As a result, the glass-water interface cannot be seen. This is an important advantage over classical confocal microscopy, as this interface produces a strong signal that masks that scattered by the sample and corrupts the 3D reconstruction. Another advantage is that the 2D angular scans in SyCM (121 illumination angles here) can be performed in a quicker way than 
the 3D position scans of standard confocal microscopy (typically more than $10^{5}$ positions required).

Next, we tested the ability of SyCM to image a biological cell. We considered human effector T lymphocytes, deposited on a glass lamella coated with adhesion molecules ICAM-1, then fixed by a treatment with paraformaldehyde at $4 \%$. Effector $\mathrm{T}$ cells migrate on ICAM-1 substrates with a strongly polarized shape. Their central and front parts are strongly adherent and spread, while their rear part forms a partially detached tail, called uropod [24].
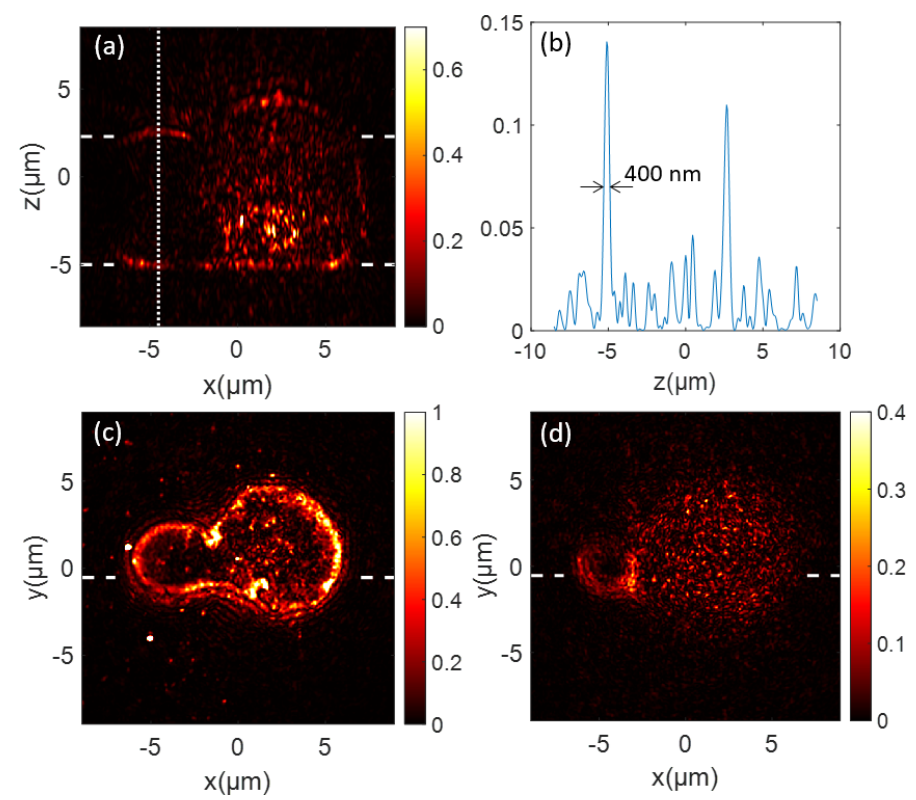

Fig. 4. Reflection SyCM intensity reconstruction of a human effector T lymphocyte: (a) axial cut along dashed green segments of (c); (b) intensity profile along vertical dotted green line of (a); (c) and (d) transverse cuts along green dashed segments of (a) at $z=-5 \mu \mathrm{m}$ (glass interface position), and at $z=2.3 \mu \mathrm{m}$, respectively. Colorscale has been readjusted on (a) and (d) for visualization purpose.

Figure 4 shows the 3D reconstruction obtained on one of such T cells: the central body on the right and the uropod on the left can be clearly identified. The central body appears filled with numerous inhomogeneities in comparison with the uropod. This can be explained by the fact that the central body contains the nucleus where highly compacted DNA is bound to produce refractive index changes stronger than anywhere else in the cell. The bottom and top membranes of the cell are also clearly retrieved on the axial cut Fig. 4(a), showing the high sectioning capability of reflection SyCM. An axial resolution about $400 \mathrm{~nm}$ can be evaluated from the full width at half maximum of the signal profile Fig. 4(b) when crossing these membranes, close to the effective wavelength of illumination of $357 \mathrm{~nm}$ in water.

\section{CONCLUSION}

In conclusion, we have shown that tomographic diffractive microscopy in reflection can be used to provide 3D images of cells that are complementary to that obtained in the more classical transmission configuration. Our TDM set-up was used as a synthetic confocal microscope in which the incident plane waves are combined numerically to form a synthetic focused beam scanning the sample. This computational approach permitted to correct easily the focus aberrations induced by the index mismatch between the oil objective $(\mathrm{NA}=1.49)$ and the mounting medium (water). It was able to image the top and bottom membrane of cells with an axial resolution about $400 \mathrm{~nm}$. This result is promising for biomedical applications where contours of biological cells have to be monitored, like for $\mathrm{T}$ cells activation detection. As a next step, the quantitative iterative reconstruction scheme used in [15] will be adapted to cells to retrieve their permittivity maps.

This project has received funding from the European Union's Marie Skłodowska-Curie grant agreement No713750, the Regional Council of Provence-Alpes-Côte d'Azur and AixMarseille University A*MIDEX ( $\mathrm{n}^{\circ}$ ANR-11-IDEX-0001-02).

Disclosures. The authors declare no conflicts of interest.

\section{REFERENCES}

1. A. Grakoui, S. K. Bromley, C. Sumen, M. M. Davis, A. S. Shaw, P. M. Allen, and M. L. Dustin, Science 285, 7 (1999).

2. V. Lauer, J. Microsc. p. 12 (2002).

3. M. Debailleul, B. Simon, V. Georges, O. Haeberlé, and V. Lauer, Meas. Sci. Technol. 19, 074009 (2008).

4. M. Debailleul, V. Georges, B. Simon, R. Morin, and O. Haeberlé, Opt. Lett. 34, 79 (2009).

5. K. Kim, K. S. Kim, H. Park, J. C. Ye, and Y. Park, Opt. Express 21, 32269 (2013).

6. U. S. Kamilov, I. N. Papadopoulos, M. H. Shoreh, A. Goy, C. Vonesch, M. Unser, and D. Psaltis, Optica 2, 517 (2015).

7. K. Lee, K. Kim, G. Kim, S. Shin, and Y. Park, Opt. Lett. 42, 999 (2017).

8. J. Lim, A. B. Ayoub, E. E. Antoine, and D. Psaltis, Light. Sci. Appl. 8 (2019).

9. A. Sentenac and J. Mertz, J. Opt. Soc. Am. A 35, 748 (2018).

10. A. Dubois, K. Grieve, G. Moneron, R. Lecaque, L. Vabre, and C. Boccara, Appl. Opt. 43, 2874 (2004).

11. O. Haeberlé, K. Belkebir, H. Giovaninni, and A. Sentenac, J. Mod. Opt. 57, 686-699 (2010).

12. S. A. Alexandrov, T. R. Hillman, T. Gutzler, and D. D. Sampson, Phys. Rev. Lett. 97 (2006).

13. V. Mico, Z. Zalevsky, P. García-Martínez, and J. García, J. Opt. Soc. Am. A 23, 3162 (2006).

14. G. Maire, Y. Ruan, T. Zhang, P. C. Chaumet, H. Giovannini, D. Sentenac, A. Talneau, K. Belkebir, and A. Sentenac, J. Opt. Soc. Am. A 30, 2133 (2013).

15. C. Godavarthi, T. Zhang, G. Maire, P. C. Chaumet, H. Giovannini, A. Talneau, K. Belkebir, and A. Sentenac, J. Opt. Soc. Am. A 32, 287 (2015).

16. H. Liu, J. Bailleul, B. Simon, M. Debailleul, B. Colicchio, and O. Haeberlé, Appl. Opt. 53, 748 (2014).

17. T. Gutzler, T. R. Hillman, S. A. Alexandrov, and D. D. Sampson, Opt. Lett. 35, 1136 (2010).

18. S. Kang, S. Jeong, W. Choi, H. Ko, T. D. Yang, J. H. Joo, J.-S. Lee, Y.-S. Lim, Q.-H. Park, and W. Choi, Nat. Photonics 9, 253-258 (2015).

19. A. Badon, D. Li, G. Lerosey, A. C. Boccara, M. Fink, and A. Aubry, SCIENCE ADVANCES p. 9 (2016).

20. Y.-Z. Liu, F. A. South, Y. Xu, P. S. Carney, and S. A. Boppart, Biomed. Opt. Express 8, 1549 (2017).

21. M. Kim, Y. Jo, J. H. Hong, S. Kim, S. Yoon, K.-D. Song, S. Kang, B. Lee, G. H. Kim, H.-C. Park, and et al., Nat. Commun. 10 (2019).

22. S. Khadir, P. C. Chaumet, G. Baffou, and A. Sentenac, J. Opt. Soc. Am. A 36, 478 (2019).

23. P. C. Chaumet, A. Sentenac, and A. Rahmani, Phys. Rev. E 70 (2004).

24. P. C. Chaumet, A. Sentenac, and T. Zhang, J. Quant. Spectrosc. Radiat. Transf. 243, 106816 (2020).

25. M.-P. Valignat, P. Nègre, S. Cadra, A. C. Lellouch, F. Gallet, S. Hénon, and O. Theodoly, Nat. Commun. 5 (2014). 


\section{REFERENCES}

1. A. Grakoui, S. K. Bromley, C. Sumen, M. M. Davis, A. S. Shaw, P. M. Allen, and M. L. Dustin, "The immunological synapse: A molecular machine controlling t cell activation," Science. 285, 7 (1999).

2. V. Lauer, "New approach to optical diffraction tomography yielding a vector equation of diffraction tomography and a novel tomographic microscope," J. Microsc. p. 12 (2002).

3. M. Debailleul, B. Simon, V. Georges, O. Haeberlé, and V. Lauer, "Holographic microscopy and diffractive microtomography of transparent samples," Meas. Sci. Technol. 19, 074009 (2008).

4. M. Debailleul, V. Georges, B. Simon, R. Morin, and O. Haeberlé, "Highresolution three-dimensional tomographic diffractive microscopy of transparent inorganic and biological samples," Opt. Lett. 34, 79 (2009).

5. K. Kim, K. S. Kim, H. Park, J. C. Ye, and Y. Park, "Real-time visualization of 3-d dynamic microscopic objects using optical diffraction tomography," Opt. Express 21, 32269 (2013).

6. U. S. Kamilov, I. N. Papadopoulos, M. H. Shoreh, A. Goy, C. Vonesch, M. Unser, and D. Psaltis, "Learning approach to optical tomography," Optica 2, 517 (2015).

7. K. Lee, K. Kim, G. Kim, S. Shin, and Y. Park, "Time-multiplexed structured illumination using a dmd for optical diffraction tomography," Opt. Lett. 42, 999 (2017).

8. J. Lim, A. B. Ayoub, E. E. Antoine, and D. Psaltis, "High-fidelity optical diffraction tomography of multiple scattering samples," Light. Sci. Appl. 8 (2019).

9. A. Sentenac and J. Mertz, "Unified description of three-dimensional optical diffraction microscopy: from transmission microscopy to optical coherence tomography: tutorial," J. Opt. Soc. Am. A 35, 748 (2018).

10. A. Dubois, K. Grieve, G. Moneron, R. Lecaque, L. Vabre, and C. Boccara, "Ultrahigh-resolution full-field optical coherence tomography," Appl. Opt. 43, 2874 (2004).

11. O. Haeberlé, K. Belkebir, H. Giovaninni, and A. Sentenac, "Tomographic diffractive microscopy: basics, techniques and perspectives," J. Mod. Opt. 57, 686-699 (2010).

12. S. A. Alexandrov, T. R. Hillman, T. Gutzler, and D. D. Sampson, "Synthetic aperture fourier holographic optical microscopy," Phys. Rev. Lett. 97 (2006).

13. V. Mico, Z. Zalevsky, P. García-Martínez, and J. García, "Synthetic aperture superresolution with multiple off-axis holograms," J. Opt. Soc. Am. A 23, 3162 (2006).

14. G. Maire, Y. Ruan, T. Zhang, P. C. Chaumet, H. Giovannini, D. Sentenac, A. Talneau, K. Belkebir, and A. Sentenac, "High-resolution tomographic diffractive microscopy in reflection configuration," J. Opt. Soc. Am. A 30, 2133 (2013).

15. C. Godavarthi, T. Zhang, G. Maire, P. C. Chaumet, H. Giovannini, A. Talneau, K. Belkebir, and A. Sentenac, "Superresolution with fullpolarized tomographic diffractive microscopy," J. Opt. Soc. Am. A 32, 287 (2015).

16. H. Liu, J. Bailleul, B. Simon, M. Debailleul, B. Colicchio, and O. Haeberlé, "Tomographic diffractive microscopy and multiview profilometry with flexible aberration correction," Appl. Opt. 53, 748 (2014).

17. T. Gutzler, T. R. Hillman, S. A. Alexandrov, and D. D. Sampson, "Coherent aperture-synthesis, wide-field, high-resolution holographic microscopy of biological tissue," Opt. Lett. 35, 1136 (2010).

18. S. Kang, S. Jeong, W. Choi, H. Ko, T. D. Yang, J. H. Joo, J.-S. Lee, Y.-S. Lim, Q.-H. Park, and W. Choi, "Imaging deep within a scattering medium using collective accumulation of single-scattered waves," Nat. Photonics 9, 253-258 (2015).

19. A. Badon, D. Li, G. Lerosey, A. C. Boccara, M. Fink, and A. Aubry, "Smart optical coherence tomography for ultra-deep imaging through highly scattering media," SCIENCE ADVANCES p. 9 (2016).

20. Y.-Z. Liu, F. A. South, Y. Xu, P. S. Carney, and S. A. Boppart, "Computational optical coherence tomography [invited]," Biomed. Opt. Express 8, 1549 (2017).

21. M. Kim, Y. Jo, J. H. Hong, S. Kim, S. Yoon, K.-D. Song, S. Kang, B. Lee, G. H. Kim, H.-C. Park, and et al., "Label-free neuroimaging in vivo using synchronous angular scanning microscopy with single-scattering accumulation algorithm," Nat. Commun. 10 (2019).
22. S. Khadir, P. C. Chaumet, G. Baffou, and A. Sentenac, "Quantitative model of the image of a radiating dipole through a microscope," J. Opt. Soc. Am. A 36, 478 (2019).

23. P. C. Chaumet, A. Sentenac, and A. Rahmani, "Coupled dipole method for scatterers with large permittivity," Phys. Rev. E 70 (2004).

24. P. C. Chaumet, A. Sentenac, and T. Zhang, "Reflection and transmission by large inhomogeneous media. validity of born, rytov and beam propagation methods," J. Quant. Spectrosc. Radiat. Transf. 243, 106816 (2020).

25. M.-P. Valignat, P. Nègre, S. Cadra, A. C. Lellouch, F. Gallet, S. Hénon, and $O$. Theodoly, "Lymphocytes can self-steer passively with wind vane uropods," Nat. Commun. 5 (2014). 